\title{
Improved Performance and Spectral Features of Complex Porous Silicon Structure Containing Silicon Solar Cells
}

\author{
E. Shatkovskis, R. Mitkevičius, V. Zagadskij and J. Stupakova \\ Vilnius Gediminas Technical University, Sauletekio all. 11, Vilnius LT 10223, Lithuania
}

\begin{abstract}
This work presents porous silicon technology, adapted to improve the characteristics of monocrystalline silicon solar cell. This is achieved by taking advantage of properties provided by porous silicon technology in production of diverse structures in the material. We produce a porous silicon derivative, which is mostly hidden in the emitter of solar cell. Research of the initial and modified solar cells was made by measuring current-voltage characteristics under illumination of a $5000 \mathrm{~K}$ xenon lamp. Spectrally resolved studies of current-voltage characteristics were carried out using radiation of halogen lamps and diffraction grating monochromator. Studies revealed that the manufacturing of buried porous silicon structure improves solar cell performance by increasing the fill factor of the modified solar cell current-voltage characteristics, maximum output power and efficiency, when compared to unmodified ones. Spectral studies revealed that the above-mentioned improvement differs for various sections of light spectrum. Largest relative enhancement of solar cell current was observed at the wavelengths of $\Delta \lambda=450-550 \mathrm{~nm}$. We consider the cumulative result of several effects resulting in solar cell efficiency enhancement. Most of them were the influence of porosity on effective optical path length and better anti-reflecting properties of multiple porous structures.
\end{abstract}

PACS: 61.43.Gt, 68.37.Hk, 72.40.+w

\section{Introduction}

Studies designed to adapt porous silicon technology to increase silicon solar cell efficiency began quite some time ago as interest in unique properties of porous silicon arose $[1,2]$. Most of research was devoted to manufacture of the porous silicon layer on the solar cell surface, reducing the reflection of incident solar radiation [3-5]. It has been shown that a layer of porous silicon results in a significant increase in energy conversion efficiency. Similar attempts to enhance the efficiency of silicon solar cells were made later $[6,7]$. It is thought that porous silicon layer may reduce the recombination rate of surface as well. It is based on analysis of a model involving carrier diffusion from the surface and recombination at the surface and in the bulk of the emitter [8]. Further review of previous work can be found in the papers $[4,9,10]$. Recently, porous silicon structure has been mostly used to enhance the efficiency of the epitaxial silicon solar cell $[11,12]$. The production of chirped Bragg reflector was performed in two steps: at first, the conventional $p$-type silicon substrate was made. Then, the $n$-type emitter was produced on a porous structure. Thus, for the first time, the porous silicon structures have been manufactured in the volume instead of the surface of the solar cell. The positive impact on solar cell efficiency was achieved by reflecting the non absorbed infrared radiation back to the $p-n$ junction. In this case, porous silicon structure improves solar cell efficiency mostly in the infrared range of the solar spectrum. Research on further increase in solar cell efficiency by devising new porous silicon structures is performed [13]. In general, most of the work observed positively impacts solar cell efficiency.

In this work, the porous silicon technology is used to improve characteristics of monocrystalline silicon solar cell by taking advantage of unique opportunity to produce diverse structures in the material [14]. We produce a combination of porous silicon derivative, whose main part is hidden in the solar cell emitter volume to improve the efficiency of silicon solar cell. The current-voltage characteristics of the cells have been measured at integral as well as at spectrally resolved illumination. Improvement of solar cell performance has been observed in samples modified by hidden porous structures. Mechanisms of the conversion efficiency enhancement and peculiarities of the spectrally resolved results have been discussed.

\section{Experiment}

Silicon solar cells under investigation were produced of $p$-type, (100) plane silicon plate by the usual silicon solar cell manufacturing technology. The surface of the plates was textured using chemical etching. The solar cell $n$-type emitter was formed using phosphorus diffusion. Continuous lower and grid upper metal contacts were made of copper by vacuum sputtering. The protective layer of the surface was made of $20-40 \mathrm{~nm}$ thick silicon nitride. The completed solar cells were $450 \mu \mathrm{m}$ thick, while the emitter thickness was about $0.5 \mu \mathrm{m}$. For the experiments, solar cell panels were cut into $5 \times 10 \mathrm{~mm}^{2}$ 
samples. The copper wire has been attached to upper and lower contacts by using the silver paste. After that, all metal contacts were protected by chemically resistant and electrically-tight sealing-wax. Manufacturing of porous silicon layer was carried out in HF:ethanol $=1: 2$ volume ratio electrolytes. The samples were illuminated by a slightly focused radiation of $50 \mathrm{~W}$ halogen lamps at the time of processing. The etching current density was computer-controlled in the limits of $5-18 \mathrm{~mA} / \mathrm{cm}^{2}$, etching time was set in the interval of $10-20 \mathrm{~s}$.

Porous silicon technology operation has been carried out in the teflon electrochemical cell. Anode was the protected lower solar cell contact, while cathode was in contact with the electrolyte made of platinum. Samples were placed in a fluoride acid for a few minutes in order to remove the protective layer of silicon nitride, before the manufacturing of porous silicon. Porous silicon structures have been produced in two stages. The low-porosity layer, about $100 \mathrm{~nm}$ thick was produced in the first stage at time period $\Delta t_{1}$. After that the current was increased - the etching was conducted at a larger current density during time period $\Delta t_{2}$. So, high-porosity layer of the structure was hidden in the emitter volume. Illumination intensity and the total etching time were chosen in such way that the porous layer was located above the solar cell $p-n$ junction [14]. At the end of the technological process, the modified solar cell sample was rinsed with ethanol and deionized water. The processing parameters of the samples are shown in Table.

Processing parameters of the samples.

TABLE

\begin{tabular}{c|c|c|c|c}
\hline \hline $\begin{array}{c}\text { Specimen } \\
\text { No. }\end{array}$ & $\begin{array}{c}J_{1} \\
{\left[\mathrm{~mA} / \mathrm{cm}^{2}\right]}\end{array}$ & $\begin{array}{c}J_{2} \\
{\left[\mathrm{~mA} / \mathrm{cm}^{2}\right]}\end{array}$ & $\begin{array}{c}\Delta t_{1} \\
{[\mathrm{~s}]}\end{array}$ & $\begin{array}{c}\Delta t_{2} \\
{[\mathrm{~s}]}\end{array}$ \\
\hline V7 & 5 & 7 & 20 & 10 \\
V8 & 5 & 10 & 20 & 10 \\
V11 & 5 & 18 & 20 & 10
\end{tabular}

Research of the initial and modified solar cells was made by measuring current-voltage characteristics illuminated by a $5000 \mathrm{~K}$ temperature radiation of xenon lamp. Spectrally resolved studies of current-voltage characteristics were carried out using radiation of halogen lamps and diffraction grating monochromator. All the electric measurements were performed using computerized equipment package consisting of multimeters Tektronix CFG 253, Keitley 2000, Metex MXD 4660, and oscilloscope Tektronix TDS 3032B.

\section{Results and discussion}

Studies revealed that manufacturing of hidden porous silicon structure improves the solar cell performance efficiency. The maximum power output in the samples V7, V8, and V11 increases by approximately 9, 20, and 61 percent, respectively, compared to samples before addition of porous silicon derivative, was observed by anal- ysis of current-voltage characteristics of the samples illuminated by xenon lamp light. As seen in Table, the samples differ only by the current density of the second etching step. Other parameters, such as the first stage current density, light intensity and etching times were fixed. Etching current density determines the etching rate and porosity of the etched layer when etching silicon electrolytically [15]. Consequently, samples V7, V8, and V11 differ only by thickness and porosity of hidden porous layer. Therefore, we observe different increase in conversion efficiency due to difference in buried porous layer thickness and their porosity.

Current-voltage characteristic measurements in different spectral regions provide the information about the properties of the hidden porous silicon layers and their impact on the solar cell efficiency. An example of the measurement data is shown in Fig. 1 for the sample V11. Only part of a total of 66 curves are shown in Fig. 1 for illustration. We have built the families of curves $P_{\max }-\lambda(U)$ at individual wavelengths $\lambda$ of the excitation radiation, for every sample including the reference (unmodified, V12) one, following usual plotting of the solar cell output power $P$ calculated from the solar cell current-voltage characteristic dependence on voltage [16]. Using these charts, the plots of the maximum solar cell output power $P_{\max }$ dependences on wavelength $\lambda$ have been found reflecting spectral dependence of the conversion efficiency.

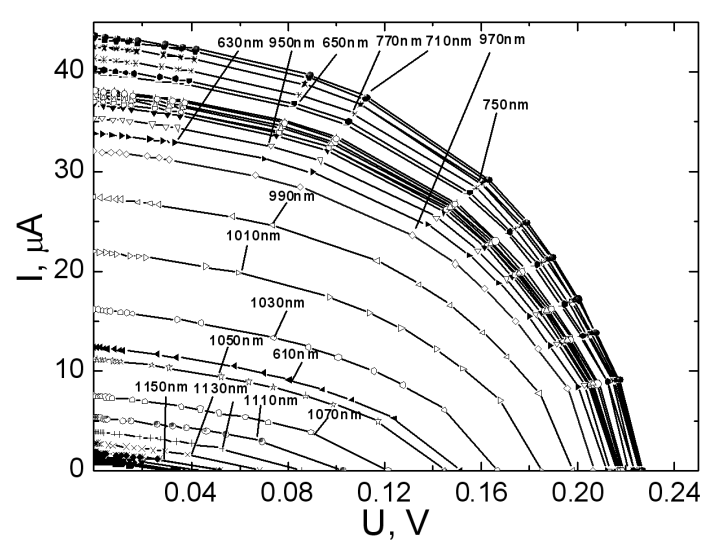

Fig. 1. Current-voltage characteristics of modified silicon solar cells illuminated by spectrally resolved light.

The curves obtained are shown in Fig. 2 for the individual modified samples as well as for reference sample V12. As shown in Fig. 2, the dependence of solar cell's maximum output power $P_{\max }$ on wavelength $\lambda$ of modified sample V7, V8, and V11 qualitatively reproduces the corresponding dependence of the reference sample V12. There is, however, no equal numerical correspondence at specific wavelengths. The generated output power changes significantly in whole spectral range $\Delta \lambda=500$ $1125 \mathrm{~nm}$. It means that the impact of hidden porous layer differs for individual samples, depending on the hidden porous layer thickness and porosity. We calculate the 


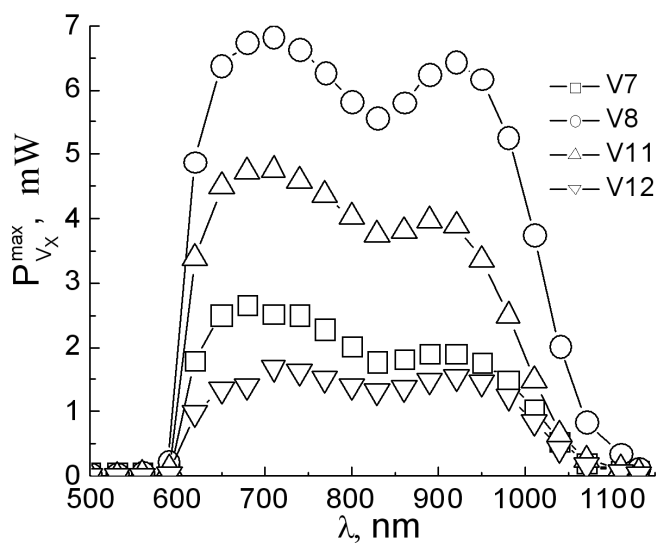

Fig. 2. Maximum power output $P^{\max }$ dependence on wavelength $\lambda$ of illuminating light in modified and unmodified silicon solar cells. Samples: V7, V8, V11 modified by hidden porous silicon structure, V12 - unmodified reference sample.

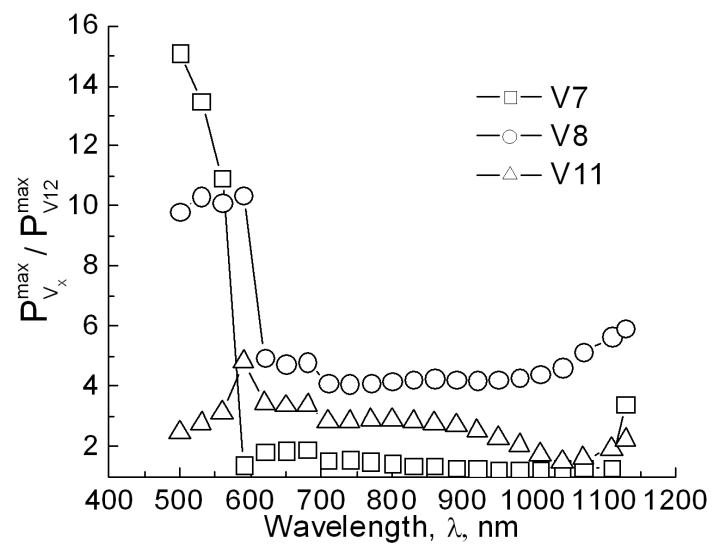

Fig. 3. Relative increase in solar cell generated power output in modified silicon solar cells versus illuminating light wavelength $\lambda$.

ratios $P_{\max } / P_{\max 0}$ at different wavelengths in order to highlight the peculiarities in spectral dependence of the light conversion efficiency.

The results of calculations are shown in Fig. 3. It is clear that the increase in generated power output of modified solar cell is uneven in different spectral regions of excitation light. The generated power output increases extremely highly in short wavelength spectral range $\Delta \lambda=500-650 \mathrm{~nm}$, whereas its value is remarkably smaller in the rest of spectral range $\Delta \lambda=650-1000 \mathrm{~nm}$. Afterwards, conversion efficiency increases to some extent again at wavelengths of $1000-1125 \mathrm{~nm}$, as seen in the figure.

Let us consider effects which may be responsible for improvement in solar cell conversion efficiencies of modified solar cells and its spectral features. We assume that this is the aggregate result of several effects. The effects which determine extremely high conversion effi- ciency gain in short-wavelength range $\Delta \lambda=500-650 \mathrm{~nm}$ will be analyzed at first. There are two plausible explanations for such result. The first one is a reduction in the surface recombination rate based on lower light absorption in porous area. Actually, in porous material, the light passes through the material, as well as the gaps. The gaps do not suffer from light loss. However, the light goes an additional distance influenced on the value of the absorption coefficient in the expression of Bouguer's law. Based on such assumption, the absorption coefficient $\alpha^{*}$ within the porous material may be written as an approximate expression

$$
\alpha^{*}=\alpha(1-A),
$$

where $\alpha$ is absorption coefficient in bulk material, $A$ denotes porosity. Therefore, the optical path length $d^{*}$ in porous material will be

$$
d^{*}=1 / \alpha^{*}=1 /[\alpha(1-A)] .
$$

The calculated optical path length in porous silicon is depicted in Fig. 4 for some wavelengths of incident light. As we can see, incident light penetrates deeper in the solar cell with porous emitter area. Deeper light penetration is particularly important in short-wavelength part of spectrum. That is, because the short-wavelength quanta are absorbed very close to the solar cell surface and the generated carriers are mainly lost due to the surface recombination. The absorption coefficient $\alpha$ is of the order of $10^{4} \mathrm{~cm}^{-1}$ in silicon at wavelength of $\approx 500 \mathrm{~nm}$, meaning that the optical path length is approximately $100 \mathrm{~nm}$. Based on applied technology, the porosity of silicon layers is about $50-75 \%$ in our samples [14]. Numerical calculation according to (2) results in an increase of optical path length by $2-4$ times at this porosity. It means that the carrier generation area of the short-wavelength quanta gets closer to the solar cell $p-n$ junction. Thus, photons take on a more favorable opportunity to participate in creating energy of the solar cell. Please note that this conclusion is compatible with results concerning reduction of surface recombination in porous silicon published in following papers [8].

Another effect which increases the conversion efficiency of modified solar cell in short-wavelength part of spectrum is possibly manifestation of selective antireflection properties of porous silicon structure. A porous silicon layer has a lower reflection and refractive indices than bulk silicon [15]. These circumstances allow the production of optical elements made of porous silicon, such as antireflection coatings and the Bragg mirrors [11, 16]. The results presented in Fig. 3 show that enhancement of solar cell power output in short-wavelength part of spectrum have the obvious optical selectivity features. It is well known that antireflection coating is made of quarter wavelength layers with different refractive indices $[3-5,16]$. We evaluate how porous silicon layers satisfy a quarter wavelength condition in our solar cells. As it is seen in Fig. 3, the greatest enhancement in efficiency of the solar cell is experienced in the vicinity of wavelengths of $550 \mathrm{~nm}$. So quarter wavelength porous layer may be 


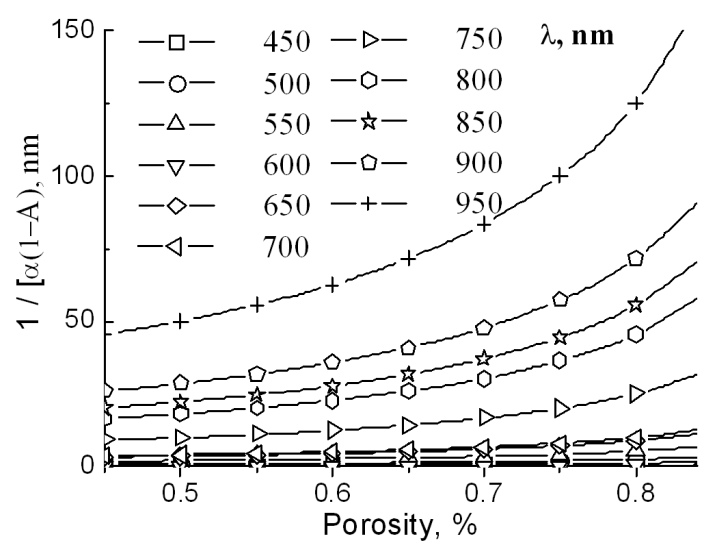

Fig. 4. Optical path length $d^{*}=1 /[\alpha(1-A)]$ dependence on porosity in the porous silicon at different wavelengths $\lambda$ with respect to material porosity.

at about $70-100 \mathrm{~nm}$ thick, taking into account that refractive index of porous silicon of porosity $A>50 \%$ is about 2 [16]. This assessment matches the porous layer thicknesses predetermined by porous silicon technology conditions used in our experiments [14].

Another effect governs the solar cell power output enhancement in middle and infrared wavelength regions of light spectrum. It is the light scattering in porous layer. Since, the incident light in porous silicon layer does not only penetrate the layer, but experiences both reflection and refraction, it results in a scattering of a light beam in greater spatial angle compared to the initial one [17]. The scattering of light at greater angle changes propagation direction lengthwise, in respect of the plane of $p-n$ junction. Thus the less absorbable light quanta are absorbed closer to the plane of the $p-n$ junction as a result. This effect called "light trapping" was systematically studied in the papers [9]. The scattering cross-section is proportional to the wavelength squared, so the "light trapping" effect gets brighter in the middle and infrared wavelength regions of light spectrum. We can also observe some additional enhancement of solar cell power output in the infrared region close to $\lambda=1100 \mathrm{~nm}$. This data fully satisfies the earlier results and confirms the conclusions made in the papers $[5,6,9]$.

As shown in Figs. 2 and 3, the solar cell specimens produced different power outputs although the illuminated area was practically identical. The enhancement of power output in respect of the reference sample is also different. As was stated above, the samples differ by hidden porous silicon layer thickness and porosity, which is caused by the difference in etching current density. These observations show that the porous silicon derivatives and their impact on solar cell efficiency can be changed by controlling the technological parameters to find the optimal effect of this option.

\section{Conclusion}

The combined porous silicon structure was manufactured in such way that its main part was hidden in the solar cell emitter volume. Spectrally resolved studies of current-voltage characteristics were carried out. It revealed that the hidden porous silicon structure essentially enhances the electrical power output generated by solar cell. The enhancement of solar cell power output is observed in wide spectral range $\Delta \lambda=500-1125 \mathrm{~nm}$. The greatest relative increase in power output was detected in the short-wavelength range $\Delta \lambda=500-650 \mathrm{~nm}$. This result was explained by short-wavelength light penetrating closer to the $p-n$ junction and by selective antireflecting impact of porous silicon derivatives. The enhancement in middle and infrared wavelength range is explained by "light trapping" effect because of light scattering in porous silicon structure.

\section{References}

[1] L. Canham, Appl. Phys. Lett. 57, 1046 (1990).

[2] V. Lehmann, U. Goesele, Appl. Phys. Lett. 58, 856 (1991).

[3] A. Prasad, S. Balakrishnan, S.K. Jain, G.C. Jain, J. Electrochem. Soc. 129, 596 (1982).

[4] P. Menna, G. Di Francia, V. La Ferrara, Sol. Energy Mater. Solar Cells 37, 13 (1995).

[5] K. Grigoras, A. Krotkus, V. Jasutis, D. Sulienè, V. Pačebutas, J. Nijs, J. Szlufcik, Lith. J. Phys. 35, 247 (1995).

[6] V. Pačebutas, K. Grigoras, A. Krotkus, Phys. Scr. 69, 255 (1997).

[7] M. Lipinski, P. Panek, S. Bastide, C. Levy-Clement, in: Porous Semicond. Science and Technology, 3d Int. Conf., Technical University of Valencia, Spain 2002, Ext. Abstr. 97.

[8] G. Kopitkovas, I. Mikulskas, K. Grigoras, I. Šimkienè, R. Tamošiūnas, Appl. Phys. A 73, 495 (2001).

[9] K. Grigoras, A. Krotkus, V. Pačebutas, I. Šimkienè, Proc. SPIE 3580, 158 (1997).

[10] V.Yu. Yerochov, I.I. Melnik, Renew. Sustain. Energy Rev. 3, 291 (1999).

[11] I. Kuzma-Filipek, F. Duerinckx, E. van Kerschaver, K. van Nieuwenhuysen, G. Beaucarne, J. Poortmans, J. Appl. Phys. 104, 073529 (2008).

[12] J. van Hoeymissen, V. Depauw, I. Kuzma-Filipek, K. van Nieuwenhuysen, M. Recaman Payo, Yu Qiu, I. Gordon, J. Poortmans, Phys. Status Solidi A 208, 1433 (2011)

[13] A. Ramizy, Z. Hassan, K. Omar, Y. Al-Douri, M.A. Mahdi, Appl. Surf. Sci. 257, 6112 (2011).

[14] E. Shatkovskis, R. Mitkevicius, V. Zagadskij, J. Stupakova, in: 4th Int. Conf. Radiation Interaction with Materials and Its Use in Technologies 2012, Kaunas (Lithuania), 2012, Technologija, Kaunas 2012, Program and Materials, p. 169.

[15] V. Lehmann, Electrochemistry of Silicon, WileyVCH, Weinheim 2002, p. 277.

[16] L. Pavesi, Riv. Nuovo Cimento 20, 1 (1997).

[17] E. Yablonovitch, G.D. Cody, IEEE Trans. Electron Dev. ED-29, 300 (1982). 